\title{
High SNR Analysis of the MIMO Interference Channel
}

\author{
Ekine Akuiyibo \\ Stanford University \\ Packard Electrical Engineering Building Faculté Informatique et Communications \\ 350 Serra Mall \\ Stanford, CA 94305, USA \\ Email: ekine@stanford.edu
}

\author{
Christophe Vignat \\ Université de Marne la Vallée \\ Institut Gaspard Monge \\ 5, Boulevard Descartes, Cité Descartes \\ 77454 Marne la Vallée Cédex 2, France \\ Email: vignat@univ-mlv.fr
}

\begin{abstract}
The rate region achievable by two transmitter-receiver pairs who wish to communicate over a Gaussian interference channel has been the subject of intense study over the last decades. Recently, the high SNR capacity region of this channel has been completely characterized in a work by Etkin, Tse and Wang (2007). In this paper, we study the effect of adding random fading into the picture, as well as multiple antennas at the transmitters and the receivers. Under the fast fading assumption, we recover a result of the same type as that obtained in the above mentioned paper. Under the slow fading assumption, we obtain an upper bound on the maximally achievable diversity order for a given target rate pair, which we conjecture to be tight.
\end{abstract}

\section{INTRODUCTION}

Considered initially by Shannon in [1], the interference channel describes the shared medium in which two or more transmitter-receiver pairs wishing to communicate reliably, interfere with each other. Although the general capacity region remains unknown in the simplest scenario with two communicating pairs, a recent result by Etkin, Tse and Wang [2] has shed light on the particular case where both interference and noise are additive, and noise is Gaussian. They establish a new outer bound on the capacity region, that is shown moreover to be achievable to within one bit/s/ $\mathrm{Hz}$ by a simplified HanKobayashi scheme [3], for all values of channel parameters. This characterization of the capacity region is therefore particularly relevant in the high SNR regime, where interference is the main factor limiting communication rates.

When transmitter-receiver pairs wish to communicate reliably in a wireless environment, users have to combat channel fading, in addition to dealing with interference. A by-now familiar technique to combat fading in the context of point-topoint communications is the use of multiple antenna systems [4]. It is therefore of interest to explore the performance achievable by such systems in the presence of interference.

We consider in this paper the situation where two transmitters are equipped with $N_{t}$ antennas each and two receivers are equipped with $N_{r}$ antennas each. In the situation where both pairs wish to communicate at the same rate, it was shown by S. A. Jafar and M. Fakhereddin in [5] that the number of degrees of freedom (or multiplexing gain) per communicating pair is given by

$$
\operatorname{dof}\left(N_{t}, N_{r}\right)=\min \left\{N_{t}, N_{r}, \frac{\max \left\{N_{t}, N_{r}\right\}}{2}\right\}
$$

Building on this result, we first exhibit, in the fast fading scenario, a rate region that characterizes the whole capacity region up to a fixed number of bits, as in [2], and provide a precise upper bound on the gap-to-capacity. Our proof technique relies on a result by [6] on a general class of interference channels. In the slow fading scenario, we analyze the maximum achievable diversity order for a given target rate pair, following the technique of [7] for point-to-point channels. Our result is an upper bound on this diversity order at high SNR. A corresponding achievability result is still lacking, but we believe that the mathematical framework required to study the upper bound is already of interest in its own right.

\section{Channel Model}

We consider the MIMO interference channel

$$
\left\{\begin{array}{l}
Y_{1}=H_{1} X_{1}+G_{1} X_{2}+Z_{1} \\
Y_{2}=G_{2} X_{1}+H_{2} X_{2}+Z_{2}
\end{array}\right.
$$

where $H_{1}, H_{2}, G_{1}, G_{2}$ are four independent $N_{r} \times N_{t}$ random matrices with i.i.d. $\mathcal{N}_{\mathbb{C}}(0,1)$ entries. These matrices are moreover independent from the $N_{r}$-variate noise vectors $Z_{1}, Z_{2}$ with i.i.d. $\mathcal{N}_{\mathbb{C}}\left(0, N_{0}\right)$ components and i.i.d realizations over time (for ease of notation, let us assume that $N_{0}=1$ ). In addition, the transmitted signals $X_{i} \in \mathbb{C}^{N_{t}}$ are subject to the (respective) power constraints $\mathbb{E}\left\{\left\|X_{i}\right\|^{2}\right\} \leq P$. For ease of notation again, let us also define the power per antenna $P_{0}=P / N_{t}$.

\section{A. Fast Fading Scenario}

We first consider the scenario where the channel matrices $H_{1}$, $H_{2}, G_{1}, G_{2}$ vary ergodically over time, and assume that the realizations of the matrices $H_{i}, G_{i}$ are revealed to receiver $i$, for $i=1,2$ respectively. That is, the received signals become

$$
\begin{aligned}
& Y_{1}=\left(H_{1} X_{1}+G_{1} X_{2}+Z_{1}, H_{1}, G_{1}\right), \\
& Y_{2}=\left(G_{2} X_{1}+H_{2} X_{2}+Z_{1}, H_{2}, G_{2}\right) .
\end{aligned}
$$


Following [6], let us also define

$$
S_{1}=\left(G_{2} X_{1}+Z_{2}, H_{2}, G_{2}\right), \quad S_{2}=\left(G_{1} X_{2}+Z_{1}, H_{1}, G_{1}\right)
$$

as well as

$$
U_{1}=\left(G_{2}^{\prime} X_{1}+Z_{2}^{\prime}, H_{2}^{\prime}, G_{2}^{\prime}\right), \quad U_{2}=\left(G_{1}^{\prime} X_{2}+Z_{1}^{\prime}, H_{1}^{\prime}, G_{1}^{\prime}\right)
$$

where $H_{1}^{\prime}, H_{2}^{\prime}, G_{1}^{\prime}, G_{2}^{\prime}, Z_{1}^{\prime}, Z_{2}^{\prime}$ are independent copies of $H_{1}, H_{2}, G_{1}, G_{2}, Z_{1}, Z_{2}$ respectively. We see that there is a one-to-one correspondence between $Y_{1}$ and $S_{2}$ given $X_{1}$, as well as between $Y_{2}$ and $S_{1}$ given $X_{2}$, and that $U_{i}$ and $S_{i}$ are independent given $X_{i}$, for $i=1,2$ respectively. The present channel therefore belongs to the general class of interference channels studied in [6]. The computation of the rate region $\mathcal{R}_{0}$ defined in there gives:

$$
\begin{aligned}
& \mathcal{R}_{0}=\left\{\left(R_{1}, R_{2}\right) \in \mathbb{R}_{+}^{2}:\right. \\
& \text { a1) } R_{1}<\mathbb{E}\left(\log \operatorname{det}\left(I+P_{0} H_{1} H_{1}^{*}\right)\right) \\
& \text { a2) } R_{2}<\mathbb{E}\left(\log \operatorname{det}\left(I+P_{0} H_{2} H_{2}^{*}\right)\right) \\
& \text { b1) } R_{1}+R_{2}<\mathbb{E}\left(\log \operatorname{det}\left(I+P_{0} H_{2} H_{2}^{*}+P_{0} G_{2} G_{2}^{*}\right)\right. \\
& \left.\quad+\log \operatorname{det}\left(I+P_{0} H_{1}\left(I+P_{0} G_{2}^{*} G_{2}^{\prime}\right)^{-1} H_{1}^{*}\right)\right) \\
& \text { b2) } R_{1}+R_{2}<\mathbb{E}\left(\log \operatorname{det}\left(I+P_{0} H_{1} H_{1}^{*}+P_{0} G_{1} G_{1}^{*}\right)\right. \\
& \left.\quad+\log \operatorname{det}\left(I+P_{0} H_{2}\left(I+P_{0} G_{1}^{*} G_{1}^{\prime}\right)^{-1} H_{2}^{*}\right)\right) \\
& \text { c1) } 2 R_{1}+R_{2}<\mathbb{E}\left(\log \operatorname{det}\left(I+P_{0} H_{1} H_{1}^{*}+P_{0} G_{1} G_{1}^{*}\right)\right. \\
& \quad+\log \operatorname{det}\left(I+P_{0} H_{1}\left(I+P_{0} G_{2}^{*} G_{2}^{\prime}\right)^{-1} H_{1}^{*}\right) \\
& \left.\quad+\log \operatorname{det}\left(I+P_{0} G_{1}^{*} G_{1}+P_{0} H_{2}^{*}\left(I+P_{0} G_{2}^{\prime} G_{2}^{\prime *}\right)^{-1} H_{2}\right)\right) \\
& c 2) R_{1}+2 R_{2}<\mathbb{E}\left(\log \operatorname{det}\left(I+P_{0} H_{2} H_{2}^{*}+P_{0} G_{2} G_{2}^{*}\right)\right. \\
& +\log \operatorname{det}\left(I+P_{0} H_{2}\left(I+P_{0} G_{1}^{* *} G_{1}^{\prime}\right)^{-1} H_{2}^{*}\right) \\
& \left.\quad+\log \operatorname{det}\left(I+P_{0} G_{2}^{*} G_{2}+P_{0} H_{1}^{*}\left(I+P_{0} G_{1}^{\prime} G_{1}^{*}\right)^{-1} H_{1}\right)\right) \\
& \text { d) } R_{1}+R_{2} \\
& \quad<\mathbb{E}\left(\log \operatorname{det}\left(I+P_{0} G_{2}^{*} G_{2}+P_{0} H_{1}^{*}\left(I+P_{0} G_{1}^{\prime} G_{1}^{* *}\right)^{-1} H_{1}\right)\right. \\
& \left.\left.\quad+\log \operatorname{det}\left(I+P_{0} G_{1}^{*} G_{1}+P_{0} H_{2}^{*}\left(I+P_{0} G_{2}^{\prime} G_{2}^{* *}\right)^{-1} H_{2}\right)\right)\right\}
\end{aligned}
$$

In [6], it is shown that $\mathcal{R}_{0}$ contains the capacity region of the above interference channel and that if $\left(R_{1}, R_{2}\right) \in \mathcal{R}_{0}$, then $\left(R_{1}-g, R_{2}-g\right)$ is achievable by a simplification of the Han-Kobayashi scheme [3], where

$$
g=I\left(X_{1} ; S_{1} \mid U_{1}\right)=I\left(X_{2} ; S_{2} \mid U_{2}\right)
$$

In the following, we compute an explicit upper bound on this gap-to-capacity $g$, in the case where the number of receive antennas is greater than or equal to the number of transmit antennas ${ }^{1}$

Proposition 1. Assume that $N_{r} \geq N_{t}$. Then the gap-tocapacity is upper bounded by

$$
g \leq \sum_{j, k=1}^{N_{t}, N_{r}} \frac{1}{N_{r}-N_{t}-1+j+k}
$$

\footnotetext{
${ }^{1}$ Notice that unlike [2], we have not been considering explictily the case where the SNR and INR scale differently, but the result of the proposition is independent of the different scalings of the SNR and INR.
}

The above result says that the gap-to-capacity is upper bounded by a fixed number, for all values of channel parameters (provided that we fix the number of antennas). It therefore provides a tight characterization of the capacity region of the fast fading MIMO interference channel in the high SNR regime, as already shown for the classical Gaussian interference channel in [2]. In particular, when $N_{r}=N_{t}=1$, the gap is upper bounded by 1 , just like for the Gaussian interference channel. Notice moreover that in this case, the high SNR capacity region is the same as that described in [2].

Also, if $N_{t}=1$, then $g \leq \sum_{k=1}^{N_{r}} \frac{1}{N_{r}-1+k} \leq 1$, for all values of $N_{r}$. If $N_{r}=N_{t}=n$, then the above bound shows that $g \leq O(n)$. At the other end, the case $N_{r}<N_{t}$ remains an open problem.

Finally, let us mention that a rapid analysis of the rate region $\mathcal{R}_{0}$ allows to recover the result (1) on the number of degrees of freedom for the MIMO interference channel when $N_{r} \geq N_{t}$.

Proof of Proposition 1. Let us compute

$$
\begin{aligned}
g= & I\left(X_{1} ; S_{1} \mid U_{1}\right)=h\left(S_{1} \mid U_{1}\right)-h\left(S_{1} \mid X_{1}, U_{1}\right) \\
= & h\left(G_{2} X_{1}+Z_{2}, G_{2}, H_{2} \mid G_{2}^{\prime} X_{1}+Z_{2}^{\prime}, G_{2}^{\prime}, H_{2}^{\prime}\right) \\
& -h\left(G_{2} X_{1}+Z_{2}, G_{2}, H_{2} \mid X_{1}, G_{2}^{\prime} X_{1}+Z_{2}^{\prime}, G_{2}^{\prime}, H_{2}^{\prime}\right) \\
= & h\left(G_{2} X_{1}+Z_{2} \mid G_{2}^{\prime} X_{1}+Z_{2}^{\prime}, G_{2}^{\prime}, G_{2}\right)-h\left(Z_{2}\right)
\end{aligned}
$$

Since $N_{r} \geq N_{t}$, the pseudo-inverse $\left(G_{2}^{\prime}\right)^{\dagger}$ of the $N_{r} \times$ $N_{t}$ matrix $G_{2}^{\prime}$ satisfies $\left(G_{2}^{\prime}\right)^{\dagger} G_{2}^{\prime}=I$, so substracting $G_{2}\left(G_{2}^{\prime}\right)^{\dagger}\left(G_{2}^{\prime} X_{1}+Z_{2}^{\prime}\right)$ to $G_{2} X_{1}+Z_{2}$ gives

$$
\begin{aligned}
g & =h\left(Z_{2}-G_{2}\left(G_{2}^{\prime}\right)^{\dagger} Z_{2}^{\prime} \mid G_{2}^{\prime} X_{1}+Z_{2}^{\prime}, G_{2}^{\prime}, G_{2}\right)-h\left(Z_{2}\right) \\
& \leq h\left(Z_{2}-G_{2}\left(G_{2}^{\prime}\right)^{\dagger} Z_{2}^{\prime} \mid G_{2}^{\prime}, G_{2}\right)-h\left(Z_{2}\right)
\end{aligned}
$$

since conditioning reduces entropy. Notice that this last inequality allows us to get rid of the input $X_{1}$ and therefore obtain a bound on the gap-to-capacity which is independent of $P$. The computation of the right-hand side leads to

$$
\begin{aligned}
g & \leq \mathbb{E}\left(\log \operatorname{det}\left(I+G_{2}\left(G_{2}^{\prime}\right)^{\dagger}\left(\left(G_{2}^{\prime}\right)^{\dagger}\right)^{*} G_{2}^{*}\right)\right) \\
& =\mathbb{E}\left(\log \operatorname{det}\left(I+G_{2}\left(G_{2}^{\prime *} G_{2}^{\prime}\right)^{-1} G_{2}^{*}\right)\right) \\
& =\mathbb{E}\left(\log \operatorname{det}\left(I+G_{2}^{*} G_{2}+G_{2}^{*} G_{2}^{\prime}\right)-\log \left(I+G_{2}^{* *} G_{2}^{\prime}\right)\right)
\end{aligned}
$$

It is now a well known fact (see e. g. [8]) that if $G$ is an $m \times n$ matrix with i.i.d. $\mathcal{N}_{\mathbb{C}}(0,1)$ entries and $m \geq n$, then

$$
\mathbb{E}\left(\log \operatorname{det}\left(I+G^{*} G\right)\right)=\sum_{j=1}^{n} \psi(m-j+1)
$$

where $\psi(m)=-\gamma+\sum_{k=1}^{m-1} \frac{1}{k}$ is the Euler digamma function. From this and the above, we deduce that

$$
\begin{aligned}
g & \leq \sum_{j=1}^{N_{t}} \psi\left(2 N_{r}-j-1\right)-\sum_{j=1}^{N_{t}} \psi\left(N_{r}-j-1\right) \\
& =\sum_{j=1}^{N_{t}}\left(\frac{1}{2 N_{r}-j}+\ldots+\frac{1}{N_{r}-j+1}\right) \\
& =\sum_{j, k=1}^{N_{t}, N_{r}} \frac{1}{N_{r}-j+k}=\sum_{j, k=1}^{N_{t}, N_{r}} \frac{1}{N_{r}-N_{t}-1+j+k}
\end{aligned}
$$


which completes the proof.

\section{B. Slow Fading Scenario}

Let us now assume that the realizations of the channel matrices $H_{1}, H_{2}, G_{1}, G_{2}$ are held fixed over time. If the channel realizations are revealed to both transmitters and receivers, it is then possible to show, following [6] again, that for a given realization of $H_{1}, H_{2}, G_{1}, G_{2}$, the capacity region $\mathcal{C}(H, G)$ of the MIMO interference channel (2) is contained in the following rate region:

$$
\mathcal{R}_{0}(H, G)=\bigcup_{\substack{Q_{1}, Q_{2} \geq 0 \\ \operatorname{Tr}\left(Q_{1}\right) \leq P, \operatorname{Tr}\left(Q_{2}\right) \leq P}} \mathcal{R}_{0}\left(H, G, Q_{1}, Q_{2}\right)
$$

where we have, for two fixed input covariance matrices $Q_{1}$ and $Q_{2}$ :

$$
\begin{aligned}
& \mathcal{R}_{0}\left(H, G, Q_{1}, Q_{2}\right)=\left\{\left(R_{1}, R_{2}\right) \in \mathbb{R}_{+}^{2}:\right. \\
& \text { a1) } R_{1}<\log \operatorname{det}\left(I+H_{1} Q_{1} H_{1}^{*}\right) \\
& \text { a2) } R_{2}<\log \operatorname{det}\left(I+H_{2} Q_{2} H_{2}^{*}\right) \\
& \text { b1) } R_{1}+R_{2}<\log \operatorname{det}\left(I+H_{2} Q_{2} H_{2}^{*}+G_{2} Q_{1} G_{2}^{*}\right) \\
& \quad+\log \operatorname{det}\left(I+H_{1} Q_{1}\left(I+Q_{1} G_{2}^{*} G_{2}\right)^{-1} H_{1}^{*}\right) \\
& \text { b2) } R_{1}+R_{2}<\log \operatorname{det}\left(I+H_{1} Q_{1} H_{1}^{*}+G_{1} Q_{2} G_{1}^{*}\right) \\
& \quad+\log \operatorname{det}\left(I+H_{2} Q_{2}\left(I+Q_{2} G_{1}^{*} G_{1}\right)^{-1} H_{2}^{*}\right) \\
& \text { c1) } 2 R_{1}+R_{2}<\log \operatorname{det}\left(I+H_{1} Q_{1} H_{1}^{*}+G_{1} Q_{2} G_{1}^{*}\right) \\
& \quad+\log \operatorname{det}\left(I+H_{1} Q_{1}\left(I+Q_{1} G_{2}^{*} G_{2}\right)^{-1} H_{1}^{*}\right) \\
& \quad+\log \operatorname{det}\left(I+Q_{2} G_{1}^{*} G_{1}+Q_{2} H_{2}^{*}\left(I+G_{2} Q_{1} G_{2}^{*}\right)^{-1} H_{2}\right) \\
& \text { c2) } R_{1}+2 R_{2}<\log \operatorname{det}\left(I+H_{2} Q_{2} H_{2}^{*}+G_{2} Q_{1} G_{2}^{*}\right) \\
& \quad+\log \operatorname{det}\left(I+H_{2} Q_{2}\left(I+Q_{2} G_{1}^{*} G_{1}\right)^{-1} H_{2}^{*}\right) \\
& \quad+\log \operatorname{det}\left(I+Q_{1} G_{2}^{*} G_{2}+Q_{1} H_{1}^{*}\left(I+G_{1} Q_{2} G_{1}^{*}\right)^{-1} H_{1}\right) \\
& \text { d) } R_{1}+R_{2} \\
& \quad<\log \operatorname{det}\left(I+Q_{1} G_{2}^{*} G_{2}+Q_{1} H_{1}^{*}\left(I+G_{1} Q_{2} G_{1}^{*}\right)^{-1} H_{1}\right) \\
& \left.\quad+\log \operatorname{det}\left(I+Q_{2} G_{1}^{*} G_{1}+Q_{2} H_{2}^{*}\left(I+G_{2} Q_{1} G_{2}^{*}\right)^{-1} H_{2}\right)\right\}
\end{aligned}
$$

Moreover, if $\left(R_{1}, R_{2}\right) \in \mathcal{R}_{0}(H, G)$, then $\left(R_{1}-N_{r}, R_{2}-\right.$ $\left.N_{r}\right) \in \mathcal{C}(H, G)$. The gap-to-capacity is therefore also upper bounded by a constant in this case, given by the number of receive antennas.

If we now assume that the realizations of the channel matrices are revealed to the receivers only, then the capacity region reduces to the single point $\{(0,0)\}$. Indeed, there is always a positive probability for a given non-zero target rate pair $\left(R_{1}, R_{2}\right)$ to fall outside the above capacity region. Relying on the above gap-to-capacity result, we analyze this outage probability in detail and establish an upper bound on the high SNR diversity-multiplexing tradeoff for the MIMO interference channel. For a target rate pair $\left(R_{1}, R_{2}\right)$, the outage probability is defined as

$$
\mathbb{P}_{\text {out }}\left(R_{1}, R_{2}\right)=\min _{\substack{Q_{1}, Q_{2} \geq 0: \\ \operatorname{Tr}\left(Q_{1}\right) \leq P, \operatorname{Tr}\left(Q_{2}\right) \leq P}} \mathbb{P}\left\{\left(R_{1}, R_{2}\right) \notin \mathcal{R}_{0}\left(Q_{1}, Q_{2}, G, H\right)\right\}
$$

and the corresponding diversity order for a target multiplexing gain pair $\left(r_{1}, r_{2}\right)$ is defined, following [7], as

$$
d\left(r_{1}, r_{2}\right)=-\lim _{P \rightarrow \infty} \frac{\log \mathbb{P}_{\text {out }}\left(r_{1} \log P, r_{2} \log P\right)}{\log P}
$$

By the above mentioned gap-to-capacity result and an argument similar to that developed in [7], it is possible to show that

$d\left(r_{1}, r_{2}\right)=-\lim _{P \rightarrow \infty} \frac{\log \mathbb{P}\left\{\left(r_{1} \log P, r_{2} \log P\right) \notin \mathcal{R}_{00}(H, G)\right\}}{\log P}$

where

$$
\begin{aligned}
& \mathcal{R}_{00}(H, G)=\left\{\left(R_{1}, R_{2}\right) \in \mathbb{R}_{+}^{2}:\right. \\
& \text { a1) } R_{1}<\log \operatorname{det}\left(I+P H_{1} H_{1}^{*}\right) \\
& a 2) R_{2}<\log \operatorname{det}\left(I+P H_{2} H_{2}^{*}\right) \\
& \text { b1) } R_{1}+R_{2}<\log \operatorname{det}\left(I+P H_{2} H_{2}^{*}+P G_{2} G_{2}^{*}\right) \\
& \quad+\log \operatorname{det}\left(I+P H_{1}\left(I+P G_{2}^{*} G_{2}\right)^{-1} H_{1}^{*}\right) \\
& \text { b2) } R_{1}+R_{2}<\log \operatorname{det}\left(I+P H_{1} H_{1}^{*}+P G_{1} G_{1}^{*}\right) \\
& \quad+\log \operatorname{det}\left(I+P H_{2}\left(I+P G_{1}^{*} G_{1}\right)^{-1} H_{2}^{*}\right) \\
& \text { c1) } 2 R_{1}+R_{2}<\log \operatorname{det}\left(I+P H_{1} H_{1}^{*}+P G_{1} G_{1}^{*}\right) \\
& \quad+\log \operatorname{det}\left(I+P H_{1}\left(I+P G_{2}^{*} G_{2}\right)^{-1} H_{1}^{*}\right) \\
& \quad+\log \operatorname{det}\left(I+P G_{1}^{*} G_{1}+P H_{2}^{*}\left(I+P G_{2} G_{2}^{*}\right)^{-1} H_{2}\right) \\
& c 2) R_{1}+2 R_{2}<\log \operatorname{det}\left(I+P H_{2} H_{2}^{*}+P G_{2} G_{2}^{*}\right) \\
& \quad+\log \operatorname{det}\left(I+P H_{2}\left(I+P G_{1}^{*} G_{1}\right)^{-1} H_{2}^{*}\right) \\
& \quad+\log \operatorname{det}\left(I+P G_{2}^{*} G_{2}+P H_{1}^{*}\left(I+P G_{1} G_{1}^{*}\right)^{-1} H_{1}\right) \\
& \text { d) } R_{1}+R_{2} \\
& \quad<\log \operatorname{det}\left(I+P G_{2}^{*} G_{2}+P H_{1}^{*}\left(I+P G_{1} G_{1}^{*}\right)^{-1} H_{1}\right) \\
& \left.\quad+\log \operatorname{det}\left(I+P G_{1}^{*} G_{1}+P H_{2}^{*}\left(I+P G_{2} G_{2}^{*}\right)^{-1} H_{2}\right)\right\}
\end{aligned}
$$

Let finally $d_{\mathrm{sym}}(r)=d(r, r)$ denote the diversity order corresponding to a symmetric target multiplexing gain, and let $d_{m, n}(r)$ denote the diversity-multiplexing tradeoff curve for a classical MIMO channel with $m$ transmit antennas, $n$ receive antennas and target multiplexing gain $r$. We recall that this curve is the polygonal line joining the points $(k, d(k)=$ $(m-k)(n-k))$ for $k$ integer between 0 and $\min (m, n)$. The following proposition gives an upper bound on the diversity order of the MIMO interference channel in the case where the number of transmit and receive antennas are equal.

Proposition 2. Assume that $N_{r}=N_{t}=n$. Then

A) $d_{s y m}(r) \leq \min \left\{d_{n, n}(r), d_{3 n, n}(2 r)\right\}$

B) $d\left(r_{1}, r_{2}\right) \leq \min \left\{d_{n, n}\left(r_{1}\right), d_{n, n}\left(r_{2}\right), d_{3 n, n}\left(r_{1}+r_{2}\right)\right\}$

We conjecture these upper bounds to be tight, but have no formal proof of this fact so far. Notice also that, as opposed to the preceding section, the above proposition does not apply to the more general situation studied in [2], where the SNR and INR scale differently; this situation would require a significantly more involved study.

Proof of Proposition 2. A) From the expression for $\mathcal{R}_{00}(H, G)$ and the fact that the matrices $H_{1}, H_{2}, G_{1}, G_{2}$ are independent 
and identically distributed, we obtain

$$
\begin{aligned}
& \mathbb{P}\left\{(r \log P, r \log P) \notin \mathcal{R}_{0}(H, G)\right\}= \\
& \mathbb{P}\{a) \log \operatorname{det}\left(I+P H_{1} H_{1}^{*}\right)<r \log P \\
& \text { or b) } \log \operatorname{det}\left(I+P H_{2} H_{2}^{*}+P G_{2} G_{2}^{*}\right) \\
& \quad+\log \operatorname{det}\left(I+P H_{1}\left(I+P G_{2}^{*} G_{2}\right)^{-1} H_{1}^{*}\right)<2 r \log P \\
& \text { or c) } \log \operatorname{det}\left(I+P H_{1}\left(I+P G_{2}^{*} G_{2}\right)^{-1} H_{1}^{*}\right) \\
& \quad+\log \operatorname{det}\left(I+P G_{1}^{*} G_{1}+P H_{2}^{*}\left(I+P G_{2} G_{2}^{*}\right)^{-1} H_{2}\right) \\
& \quad+\log \operatorname{det}\left(I+P H_{1} H_{1}^{*}+P G_{1} G_{1}^{*}\right)<3 r \log P \\
& \text { or } d) \log \operatorname{det}\left(I+P G_{2}^{*} G_{2}+P H_{1}^{*}\left(I+P G_{1} G_{1}^{*}\right)^{-1} H_{1}\right) \\
& \quad+\log \operatorname{det}\left(I+P G_{1}^{*} G_{1}+P H_{2}^{*}\left(I+P G_{2} G_{2}^{*}\right)^{-1} H_{2}\right) \\
& \quad<2 r \log P\}
\end{aligned}
$$

Any of the four bounds above imposes an upper limit on the diversity order. In particular, it follows directly from [7] that the single-user bound a) leads to the upper bound

$$
d_{\text {sym }}(r) \leq d_{n, n}(r)
$$

where $d_{n, n}(r)$ is the classical $n \times n$ MIMO diversity curve.

Let us now analyze the limitation imposed by bound b) in (3). We easily see that

$$
\begin{aligned}
& \log \operatorname{det}\left(I+P H_{2} H_{2}^{*}+P G_{2} G_{2}^{*}\right) \\
& +\log \operatorname{det}\left(I+P H_{1}\left(I+P G_{2}^{*} G_{2}\right)^{-1} H_{1}^{*}\right) \\
= & \log \operatorname{det}\left(I+P H_{2} H_{2}^{*}+P G_{2} G_{2}^{*}\right)-\log \operatorname{det}\left(I+P G_{2}^{*} G_{2}\right) \\
& +\log \operatorname{det}\left(I+P H_{1}^{*} H_{1}+P G_{2}^{*} G_{2}\right)
\end{aligned}
$$

It is a well know fact (see e. g. [9]) that if $G$ is a $n \times n$ matrix with i.i.d. $\mathcal{N}_{\mathbb{C}}(0,1)$ entries, then $G G^{*}=U \Lambda U^{*}$, where $\Lambda$ is a diagonal matrix formed by the (ordered) eigenvalues of $G G^{*}, U$ is uniformly distributed on the set of $n \times n$ unitary matrices and $\Lambda$ and $U$ are independent. Likewise, $G^{*} G=$ $V \Lambda V^{*}$, where $V$ is a uniformly distributed unitary matrix, also independent of $\Lambda$. Therefore, the above expression is still equal to

$\log \operatorname{det}\left(I+P W_{2} M_{2} W_{2}^{*}+P U_{2} \Lambda U_{2}^{*}\right)-\log \operatorname{det}\left(I+P V_{2} \Lambda V_{2}^{*}\right)$ $+\log \operatorname{det}\left(I+P W_{1} M_{1} W_{1}^{*}+P V_{2} \Lambda V_{2}^{*}\right)$

where $\Lambda, M_{1}, M_{2}$ are diagonal, $U_{2}, V_{2}, W_{1}, W_{2}$ are uniformly distributed unitary matrices and all matrices, except $U_{2}$ and $V_{2}$, are independent. Using the identity $\operatorname{det}(I+A B)=\operatorname{det}(I+$ $B A)$, the above expression may be rewritten as

$$
\begin{aligned}
& \log \operatorname{det}\left(I+P\left(U_{2}^{*} W_{2}\right) M_{2}\left(W_{2}^{*} U_{2}\right)+P \Lambda\right)-\log \operatorname{det}(I+P \Lambda) \\
& +\log \operatorname{det}\left(I+P\left(V_{2}^{*} W_{1}\right) M_{1}\left(W_{1}^{*} V_{2}\right)+P \Lambda\right)
\end{aligned}
$$

Now, since $U_{2}, W_{2}, V_{2}, W_{1}$ are uniformly distributed unitary matrices, so are $U_{2}^{*} W_{2}$ and $V_{2}^{*} W_{1}$ (and these two are independent); therefore, $\left(U_{2}^{*} W_{2}\right) M_{2}\left(W_{2}^{*} U_{2}\right)$ has the same distribution as $H_{2} H_{2}^{*}$ and $\left(V_{2}^{*} W_{1}\right) M_{1}\left(W_{1}^{*} V_{2}\right)$ has the same distribution as $H_{1}^{*} H_{1}$ (or equivalently $H_{1} H_{1}^{*}$ ), which in turn implies that (4) has the same distribution as

$$
\begin{aligned}
& \log \operatorname{det}\left(I+P H_{2} H_{2}^{*}+P \Lambda\right)-\log \operatorname{det}(I+P \Lambda) \\
& +\log \operatorname{det}\left(I+P H_{1} H_{1}^{*}+P \Lambda\right)
\end{aligned}
$$

Using then Hadamard's inequality, we obtain that the above expression is less than or equal to

$$
\begin{aligned}
\sum_{j=1}^{n}\{ & \log \left(1+P\left(\left\|h_{j}^{(2)}\right\|^{2}+\lambda_{j}\right)\right)-\log \left(1+P \lambda_{j}\right) \\
& \left.+\log \left(1+P\left(\left\|h_{j}^{(1)}\right\|^{2}+\lambda_{j}\right)\right)\right\}
\end{aligned}
$$

where $\lambda_{1} \geq \ldots \geq \lambda_{n}$ are the eigenvalues of $G_{2} G_{2}^{*}$ and $h_{j}^{(i)}$ is the $j^{\text {th }}$ row of $H_{i}$.

To summarize, what we have shown so far is that

$$
\begin{aligned}
\mathbb{P}\left\{(r \log P, r \log P) \notin \mathcal{R}_{00}(H, G)\right\} \\
\geq \mathbb{P}\left\{\log \operatorname{det}\left(I+P H_{2} H_{2}^{*}+P G_{2} G_{2}^{*}\right)\right. \\
\left.\quad+\log \operatorname{det}\left(I+P H_{1}\left(I+P G_{2}^{*} G_{2}\right)^{-1} H_{1}^{*}\right)<2 r \log P\right\} \\
\geq \mathbb{P}\left\{\sum _ { j = 1 } ^ { n } \left\{\log \left(1+P\left(\left\|h_{j}^{(2)}\right\|^{2}+\lambda_{j}\right)\right)-\log \left(1+P \lambda_{j}\right)\right.\right. \\
\left.\left.+\log \left(1+P\left(\left\|h_{j}^{(1)}\right\|^{2}+\lambda_{j}\right)\right)\right\}<2 r \log P\right\}
\end{aligned}
$$

The joint distribution of $\lambda_{1} \geq \ldots \geq \lambda_{n}$ is the classical Wishart distribution

$$
p\left(\lambda_{1}, \ldots, \lambda_{n}\right) \sim \prod_{j<k}\left(\lambda_{k}-\lambda_{j}\right)^{2} \exp \left(-\sum_{j=1}^{n} \lambda_{j}\right)
$$

and the norms $B_{j}^{(i)}=\left\|h_{j}^{(i)}\right\|^{2}$ are i.i.d. random variables (independent of the $\lambda$ 's), each with Gamma distribution

$$
p(B) \sim B^{n-1} \exp (-B)
$$

Following the methodology of [7], let us make the change of variables

$$
\lambda_{j}=P^{-\alpha_{j}}, \quad B_{j}^{(i)}=P^{-\beta_{j}^{(i)}}
$$

It is then possible to deduce the following upper bound on the diversity order:

$$
d_{\text {sym }}(r) \leq \min \sum_{j=1}^{n}\left\{(2(n-j)+1) \alpha_{j}+n\left(\beta_{j}^{(1)}+\beta_{j}^{(2)}\right)\right\}
$$

where the minimization over $\alpha$ 's and $\beta$ 's is subject to the constraints:

$$
\alpha_{n} \geq \ldots \geq \alpha_{1} \geq 0, \quad \beta_{j}^{(i)} \geq 0
$$

and

$$
\begin{gathered}
\sum_{j=1}^{n}\left\{\max \left(0,1-\alpha_{j}, 1-\beta_{j}^{(1)}\right)+\max \left(0,1-\alpha_{j}, 1-\beta_{j}^{(2)}\right)\right. \\
\left.-\left(1-\alpha_{j}\right)^{+}\right\}<2 r
\end{gathered}
$$

Notice that by symmetry, this upper bound may be simplified to

$$
d_{\mathrm{sym}}(r) \leq \min \sum_{j=1}^{n}\left\{(2(n-j)+1) \alpha_{j}+2 n \beta_{j}\right\}
$$

subject to $\alpha_{n} \geq \ldots \geq \alpha_{1} \geq 0, \beta_{j} \geq 0$ and

$$
\sum_{j=1}^{n}\left\{2 \max \left(0,1-\alpha_{j}, 1-\beta_{j}\right)-\left(1-\alpha_{j}\right)^{+}\right\}<2 r
$$


This minimization problem has the following elegant solution: for $2 r=k$ integer between 0 and $n$, we have $\alpha_{j}^{*}=\beta_{j}^{*}=0$ for $j<=k$ and $\alpha_{j}^{*}=\beta_{j}^{*}=1$ for $j>k$, so that

$d_{\text {sym }}(k / 2) \leq \sum_{j=k+1}^{n}\{(2(n-j)+1)+2 n\}=(3 n-k)(n-k)$

and for non-integer values of $2 r$, the curve is a linear interpolation between these points. This turns out to be the classical $3 n \times n$ MIMO diversity curve (scaled horizontally by a factor $1 / 2)$, so that

$$
d_{\text {sym }}(r) \leq d_{3 n, n}(2 r)
$$

This completes the proof of part $\mathrm{A})^{2}$.

B) The second part of the proposition follows from a straightforward extension of the above argument.

\section{Illustrations of the result:}

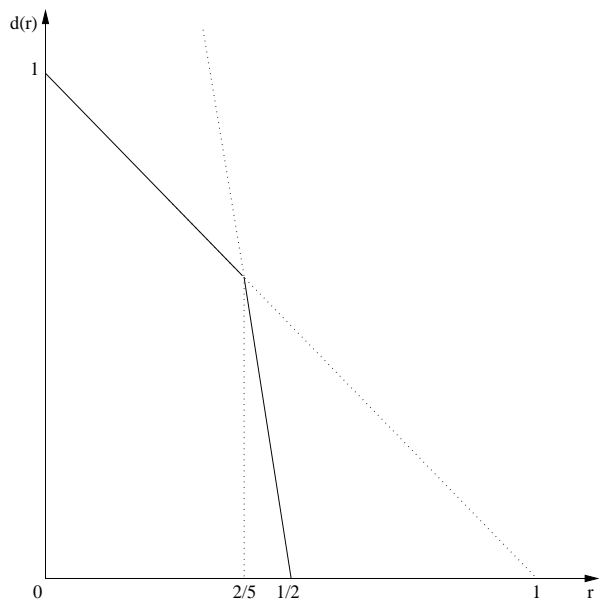

Fig. 1: upper bound on the diversity order $d_{\text {sym }}(r)$ for $n=1$.

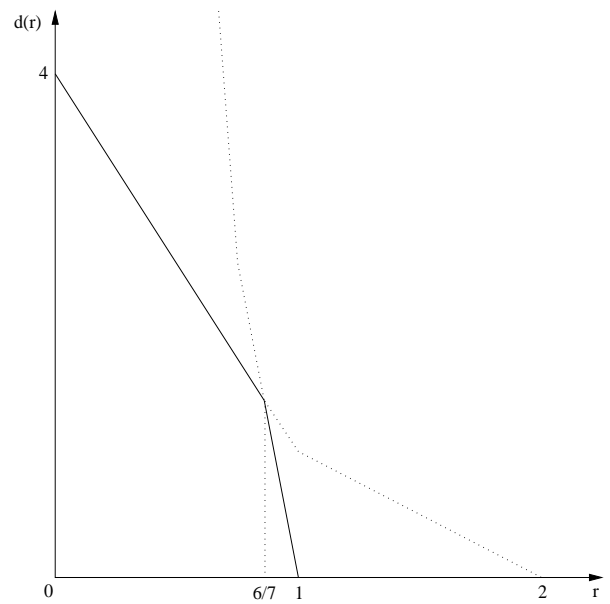

Fig. 2: upper bound on the diversity order $d_{\text {sym }}(r)$ for $n=2$.

\footnotetext{
${ }^{2}$ We conjecture that neither bound c) nor bound d) impose stricter limitations on the diversity order than those imposed by bounds a) and b) together. This fact is however not needed in order to state the proposition.
}

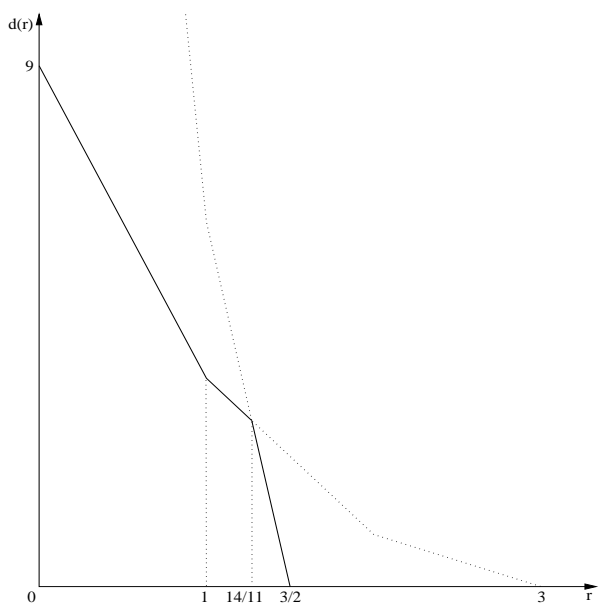

Fig. 3: upper bound on the diversity order $d_{\mathrm{sym}}(r)$ for $n=3$.

Finally, we make the following conjecture, in the case where the number of receive antennas is not equal to the number of transmit antennas. This conjecture is in agreement with the number of degrees of freedom (1) of the MIMO interference channel. Notice however that a straightforward extension of the above argument does not suffice to prove the conjecture.

Conjecture 3. Let $m=\max \left(N_{r}, N_{t}\right)$ and $n=\min \left(N_{r}, N_{t}\right)$. Then

$$
\begin{aligned}
& \text { A) } \begin{aligned}
& d_{\text {sym }}(r) \leq \min \left\{d_{a}(r), d_{b}(r)\right\} \\
& \text { where } d_{a}(r)=d_{m, n}(r) \\
& \qquad \begin{aligned}
d_{b}(r) & =d_{2 m, n}(2 r)+d_{m, 2 n}(2 r)-d_{m, n}(2 r) \\
& =d_{m, 2 n}(2 r)+m(n-2 r)^{+}
\end{aligned} \\
& \text {B) } d\left(r_{1}, r_{2}\right) \leq \min \left\{d_{a}\left(r_{1}\right), d_{a}\left(r_{2}\right), d_{b}\left(r_{1}+r_{2}\right)\right\}
\end{aligned}
\end{aligned}
$$

\section{ACKNOWLEDGMENT}

We would like to thank Emre Telatar for helpful discussions.

\section{REFERENCES}

[1] C. E. Shannon, Two-Way Communication Channels, Proc. 4th Berkeley Symposium on Mathematical Statistics and Probability 1, Berkeley, CA, Univ. California Press, 611-644, 1961.

[2] R. Etkin, D. Tse, H. Wang, Gaussian Interference Channel Capacity to Within One Bit, submitted to the IEEE Transactions on Information Theory, February 2007.

[3] T. S. Han and K. Kobayashi, A New Achievable Rate Region for the Interference Channel, IEEE Transactions on Information Theory 27 (1), 49-60, January 1981.

[4] E. Telatar, Capacity of Multi-antenna Gaussian Channels, European Transactions on Telecommunications 10 (6), 585-596, 1999.

[5] S. A. Jafar, M. J. Fakhereddin, Degrees of Freedom for the MIMO Interference Channel, IEEE Transactions on Information Theory 53 (7), 2637-2642, July 2007,

[6] E. Telatar, D. Tse, Bounds on the Capacity Region of a Class of Interference Channels, Proceedings of the IEEE Symposium on Information Theory, Nice France, June 2007.

[7] L. Zheng, D. Tse, Diversity and Multiplexing: A Fundamental Tradeoff in Multiple-Antenna Channels, IEEE Transactions on Information Theory 49 (5), 1073-1096, May 2003.

[8] A. Lozano, A. M. Tulino, S. Verdú: High-SNR Power Offset in Multiantenna Communication, IEEE Transactions on Information Theory 51 (12), 4134-4151, December 2005.

[9] M. L. Mehta, Random matrices. Third edition. Academic Press, Inc., Boston, MA, 2004. 